\title{
Mitigation of Head and Neck Cancer Service Disruption During COVID-19 in Hong Kong Through Telehealth and Multi-institution Collaboration
}

\author{
Zenon WC Yeung, FRCSEd (ORL) ${ }^{1}$ \\ ${ }^{1}$ United Christian Hospital and Tseung Kwan O Hospital
}

April 28, 2020

\begin{abstract}
The 2019 novel coronavirus disease (COVID-19) pandemic has been spreading worldwide at an alarming rate. Healthcare workers have been confronted with the challenge of not only treating patients with the virus, but also managing the disruption of healthcare services caused by COVID-19. In anticipation of outbreak, clinic sessions and operation theatre lists have been actively cut back since February 2020 to reduce hospital admissions and clinic attendances. This has severely disrupted healthcare services, leading to accumulating clinic caseload and substantial delays for operations. The head and neck cancer service has been faced with the difficult task of managing the balance between infection risk to healthcare providers and the risk of disease progression from prolonged waiting times. We share our experience in Hong Kong on the mitigation of head and neck cancer service disruption through telehealth and multi-institution collaboration.
\end{abstract}

\section{Authors}

${ }^{1}$ Alex KF Lee MRCS, ${ }^{1}$ Ryan HW Cho FRCSEd (ORL), ${ }^{2}$ Eric HL Lau FRCSEd (ORL), ${ }^{3}$ HK Cheng MBBS, FANZCA, FHKAM, ${ }^{2}$ Eddy WY Wong FRCSEd (ORL), ${ }^{1}$ Peter KM Ku FRCSEd (ORL), ${ }^{2}$ Jason YK Chan FRCSEd (ORL), ${ }^{1}$ Zenon WC Yeung FRCSEd (ORL)

\section{Author Affiliations}

${ }^{1}$ Department of Otorhinolaryngology - Head and Neck Surgery, United Christian

Hospital and Tseung Kwan O Hospital, Hong Kong

${ }^{2}$ Department of Otorhinolaryngology - Head and Neck Surgery, The Chinese University of Hong Kong, Hong Kong

${ }^{3}$ Department of Anesthesiology, Tseung Kwan O Hospital, Hong Kong

Corresponding Authors

Zenon WC Yeung, FRCSEd (ORL)

Department of Otorhinolaryngology — Head and Neck Surgery,

United Christian Hospital and Tseung Kwan O Hospital

2 Po Ning Lane, Tseung Kwan O

New Territories

Hong Kong 
Email: dr.zenon.yeung@gmail.com

Phone: 85292734534

Jason YK Chan, FRCSEd (ORL)

Department of Otorhinolaryngology, Head and Neck Surgery

The Chinese University of Hong Kong

Room 84026, 6/F, Lui Che Woo Clinical Sciences Building, Prince of Wales Hospital, Shatin New Territories

Hong Kong

Email: jasonchan@ent.cuhk.edu.hk

Phone: +852 97081554

\title{
Keywords:
}

Head and neck, otolaryngology, Hong Kong, COVID-19, telehealth

\begin{abstract}
The 2019 novel coronavirus disease (COVID-19) pandemic has been spreading worldwide at an alarming rate. Healthcare workers have been confronted with the challenge of not only treating patients with the virus, but also managing the disruption of healthcare services caused by COVID-19. In anticipation of outbreak, clinic sessions and operation theatre lists have been actively cut back since February 2020 to reduce hospital admissions and clinic attendances. This has severely disrupted healthcare services, leading to accumulating clinic caseload and substantial delays for operations. The head and neck cancer service has been faced with the difficult task of managing the balance between infection risk to healthcare providers and the risk of disease progression from prolonged waiting times. We share our experience in Hong Kong on the mitigation of head and neck cancer service disruption through telehealth and multi-institution collaboration.
\end{abstract}

\section{Introduction}

The 2019 novel coronavirus disease (COVID-19) pandemic has affected millions of people worldwide and paralyzed the health care systems in different major countries since its onset. COVID-19 originated in Wuhan, China in December 2019(1-3), the largest and most populous city in central China, and a major regional transportation hub. The Hong Kong SAR, being the most densely populated city in southern China and a major transport hub connecting China to the rest of the world, faced the first confirmed coronavirus case on 23rd January 2020 imported from Mainland China. The initial confirmed cases were all imported from Mainland China, followed later by evidence of community spread with local transmission on the 2nd February 2020. The number of cases in Hong Kong rapidly increased in March 2020, with a large proportion of cases being imported from Western Countries, and as of April 14, there were 1013 confirmed cases(4). During this pandemic, in anticipation of rapid growth in confirmed cases yet limited resources in the public health care system, there was a tremendous pressure on reducing hospital admissions and clinic attendances since early February 2020. Here we outline the changes in resource allocations and its effect on our clinical and operating service provision.

\section{Clinic Arrangement and Telehealth}

To minimize the possibility of cross contamination and nosocomial infections within hospitals, the majority of routine clinic appointments were postponed(5). 80-90\% of outpatient service have been cut to leave only 
urgent or semi-urgent conditions to be seen. During this two-month period, 3800 cases have been rescheduled at the outpatient clinic. The number of doctors seeing consultations was reduced significantly, with one dedicated clinician with full personal protective equipment(6) and fit tested N95 respirator performing aerosol generating procedures such as a flexible laryngoscopy, to conserve personal protective equipment (PPE) during clinics. In addition, each doctor sees only 10-15 patients per consultation session, representing a reduction of caseload by at least $40 \%$ for each doctor, allowing the time needed for strict compliance of infection control measures. All doctors wore a gown, cap, gloves and surgical mask to see patients(5) and all patients were required to wear a surgical mask before entering the hospital. A dedicated drug refill clinic was also set up to allow stable patients to obtain a repeat prescription without consultation and new appointments were rescheduled based on their clinical condition.

To deal with the accumulation of rescheduled cases, the department may need to set up extra clinic sessions in the evening or during weekends once the pandemic is settled. It will take about 44 weeks with an average of 2 extra sessions per week to tackle the accumulated cases. In order to avoid excessive rescheduling of appointments, our hospital has started utilizing tele-medicine in clinical practice during the pandemic since February 2020. 2 sessions were organized per week for each doctor with 6 consultations per session. Telemedicine sessions took up to 10 minutes longer when compared to a face to face consultation of usually less than 10 minutes per patient.

To accomplish these telemedical sessions, Zoom was adopted by the hospital as an app in compliance with Health Insurance Portability and Accountability Act Security Standards to ensure the security and privacy of protected health information. This platform for telemedicine was endorsed by the Central Credentialing Committee, Hospital Authority, Hong Kong. The spectrum of telemedicine service included consultations by doctors and nurses, outpatient wound care, palliative care at home and academic meetings or multidisciplinary tumor board meetings. However, there have been recent concerns over the potential vulnerability with Zoom, in which 'Zoombombing' or hijacking during video conferencing(7), and privacy concerns have been raised. The Hospital Authority in Hong Kong underwent a series of security assessments on the updated Zoom client, confirming that data sharing with social media was no longer allowed. The analysis reported that the vulnerabilities have been resolved and end-to-end encryption was also enforced. To protect our patients, the Information Technology department of the Hospital Authority recommended clinicians and patients to use the latest version of the Zoom client and to use the Zoom client with a corporate account to enjoy enterprise security features. Furthermore, a password should be set for every meeting and the meeting details and password should be exclusively disclosed to participants only. Finally, the "Lock" function should be enabled once all participants have joined the meeting to avoid intrusion.

The key to the successful operation of telemedicine consultations was patient selection.

Ideal cases would be patients with routine follow-up whom physical examination might not add critical information. Patients' age and education were also taken into consideration. Patients who were enrolled into telehealth had to possess a smart device with a web camera and be able to use the Zoom web conferencing app. In head and neck patients, telemedicine could be utilized to reduce patient's travel and hospital attendance to minimize the risk of cross infection. After the initial attendance for examination and consent, further preoperative counselling, education of postoperative care, dietary advice and neck and shoulder exercise recommendations could all be delivered by telemedicine. In the postoperative phase, simple wound review and troubleshooting could also be performed.

To ensure the correct patient was seen at the right time using Zoom tele-conference, the appointment date and time, Zoom conference ID and password were sent to patient's email and phone using SMS. Two-way ID verification was then adopted. Patients were asked about their date of birth or the last 3 digits of their Hong Kong identity card number and they were asked to show their unique outpatient appointment slip to the web camera. After each consultation, a new appointment slip and prescription sheet would be mailed to the patient by post. At the same time, an electronic appointment slip and prescription would be sent to patients through e-mail or the hospital telehealth app. Patients could then go to the Pharmacy within 7 days to obtain the prescribed medications. (Figure 1) 


\section{Operation Theatre arrangement}

Amidst the rapidly evolving outbreak of COVID-19, there have been growing concerns of the climbing caseload, cross infection, safety and nosocomial infection for patients and healthcare workers alike.

Gleaning from the experience from managing patients during the SARS epidemic in 2003, hospitals in Hong Kong began to introduce measures in the operation theatre to protect healthcare workers and patients and in anticipation of outbreak since February 2020.

\section{Perioperative protective measures}

Full sets of PPE including cap, gown, N95 particulate respirator, and gloves were supplied to all essential staff during aerosol generating procedures, including during intubation and extubation and for airway operations.

To reduce contact and conserve PPE, personnel were kept to a minimum perioperatively for such procedures. During intubation and extubation, only the anesthetists and other essential staff remained in the operation room, with the surgeons and operating nurses on standby outside. Anesthetists used videolaryngoscopes with a transparent plastic drape to form a closed circuit and a barrier between them and the patient's airway as an extra protective measure for decreasing aerosol spread and contact during intubation and extubation. Only experienced surgeons were selected for airway operations such as tracheostomies in order to reduce potential risk and total contact time.

\section{Reduction of operation theatre service}

Due to infection concerns, anticipated need for redistribution of manpower and reservation of hospital beds for potential infectious cases, operation theatre services have been stripped to emergency and priority elective operations since February 2020, including head and neck cancer surgeries.

Whilst vital in combating the spread of the virus, this invariably introduces inconvenience to patients and runs the risk of delayed assessment and surgery. This is particularly relevant to head and neck cancer patients, where longer waiting time could potentially lead to disease progression and altered prognosis.

The dilemma of balancing between infection concerns from COVID-19 and ensuring appropriate surgical management has been shared by head and neck surgeons all over the world. Several guidelines have been published to prioritize the otolaryngologic and head and neck procedures and to postpone elective procedures during the pandemic $(8,9)$. The Center for Medicaid and Medicare Services in the US has recommended all elective surgeries, non-essential medical, surgical, and dental procedures be delayed during the pandemic. In the United Kingdom, it was recommended during this contingency period to prioritise day case surgery and restriction or cessation of surgical procedures requiring admission to intensive care unit post-operatively. Suggestions have also made to reduce the length of surgery by use of local or pedicled flaps rather than free flaps $(9)$.

For patients in the Hong Kong public healthcare system, they are referred to our head and neck center where an initial assessment is made. Investigations and operations are then scheduled into a public queue. Prior to the COVID-19 pandemic, the waiting time from point of diagnosis to surgery in our head and neck center was an average of 4 weeks, with 6 dedicated operating lists per month. With the reduction in operation theatre service, head and neck elective operation sessions have been decreased to a third of its original number, and with an ongoing influx of new cases, the waiting time has been lengthened to as long as 6 months.

To confront the challenge of limited resources and reduced operating lists, our head and neck center stratified operations into 3 tiers of priority with 3 targeted waiting times (Figure 2). They are divided according to the aggressiveness of the tumor, potential effect on reconstruction and anticipated complications. With this approach, we aim to limit the disruption to patient care as much as possible.

Squamous cell carcinomas were prioritized due to its high tumor volume doubling time and rapid disease progression, with prior studies showing TNM progression and new lymph node metastases within a 4-week waiting time(10). High-grade salivary gland tumors were also given high priority due to their aggressive 
clinical behavior and relatively favorable prognosis in early stages of disease(11). Tumors with more indolent behavior such as papillary thyroid carcinomas or low-grade salivary gland tumors were given a relatively lower priority.

Early operations were performed for small oral cavity tumors in order to avoid the potentially more complex reconstruction with delayed surgery, which may introduce higher morbidity and prolonged hospital stay for the patient. Similarly, bulky tumors of the larynx and hypopharynx were also prioritized to avoid the possibility of progression to airway obstruction requiring an emergency tracheostomy.

In order to achieve the targeted waiting times, our head and neck department has initiated a collaboration with other ENT units across Hong Kong to arrange early surgery for more urgent cases in different hospitals. Tier 1 or 2 patients with an anticipated waiting time beyond our target were prioritized, and to ensure appropriate perioperative care, ENT units with head and neck expertise and intensive care units were selected. A full explanation was given to each patient and if agreed, they were referred to the partner ENT unit for a preoperative consultation, then for an earlier surgery. Prior to each referral, there was extensive communication between the two units to ensure a consensus for the surgical plan and that an operating list was available.

The distribution of priority head and neck cancer patients between regional ENT units to provide timely surgery was made possible due to the proximity of hospitals in Hong Kong and the close-knit local Head and Neck community.

Since the initiation of this multi-institution collaboration in March, we have been able to alleviate the number of pending head and neck cancer operations by $20 \%$. We hope to expand this cooperation in the coming months to further mitigate the accumulating caseload and obtain timely surgery for our patients.

\section{Discussion}

COVID-19 has spread across the world, involving 213 countries with at least 1.8 million confirmed cases and 117,021 confirmed deaths as of Apr 14, 2020(12). Evolving data from China and Italy has shown that nosocomial infection occurred when there was insufficient protection. 1716 cases of nosocomial infections have been reported in China including 5 mortalities(13). The fact that a significant proportion of asymptomatic carriers continue to shed virus during the incubation period poses increased risk to health care professionals(8). It is therefore of paramount importance to ensure adequate protection for health care workers and to prevent the spread of the virus by providing suitable protective equipment and limiting clinic and operation sessions. Otolaryngologists have been known to be of notable risk because of the need to examine the upper aerodigestive tract, which carries the highest viral load, particularly in the nose and throat $(8,9)$.

It has been encouraging to see no single case of nosocomial infection in Hong Kong

despite more than 1000 confirmed cases of COVID-19. This confirms that the current practice of infection control is successful in preventing nosocomial infection.

However, these efforts to contain the spread of the virus has given rise to issues of accumulating caseload in the clinics and operating lists and diminishing personal protective equipment supplies. Head and neck surgeons have been faced with the unique challenge of balancing infection risk and appropriately timed surgery due to the risk of disease progression in head and neck cancers. Faced with these challenges, extensive efforts have been made both in the clinics and operation theatre services to minimize the disruption to usual healthcare services. Novel methods such as telemedicine and regional collaboration with other ENT centers have been implemented with some success, with plans for further development in the coming months.

Despite all the measures taken, a substantial number of patients remain greatly affected. Lower priority surgeries such as those for low grade malignancies and benign head and neck tumors have been postponed and will require resolution as soon as the pandemic settled.

\section{References}


1. Wang D, Hu B, Hu C et al. Clinical Characteristics of 138 Hospitalized Patients With 2019 Novel Coronavirus-Infected Pneumonia in Wuhan, China. JAMA. 2020.

2. Novel Coronavirus Pneumonia Emergency Response Epidemiology T. The epidemiological characteristics of an outbreak of 2019 novel coronavirus diseases (COVID-19) in China. Zhonghua Liu Xing Bing Xue Za Zhi. 2020;41(2):145-51.

3. Khan S, Nabi G, Han G et al. Novel coronavirus: how things are in Wuhan. Clin Microbiol Infect. 2020.

4. SAR TGoHK. Coronavirus Disease (COVID-19) in HK. 2020.

5. Chan JYK, Wong EWY, Lam W. Practical Aspects of Otolaryngologic Clinical Services During the 2019 Novel Coronavirus Epidemic: An Experience in Hong Kong. JAMA Otolaryngol Head Neck Surg. 2020.

6. (HKCORL) THKCoO. The Guide to the Proper Techniques for Gowning and Removal of Personal Protective Equipment (PPE) 2020 [Available from: https://hkcorl.org.hk/database/files/hkcorl_video_20200327.mp4.

7. Toronto TCLUo. A Quick Look at the Confidentiality of Zoom Meetings 2020 [Available from: https://citizenlab.ca/2020/0 fast-roll-your-own-crypto-a-quick-look-at-the-confidentiality-of-zoom-meetings/.

8. Zou L, Ruan F, Huang M et al. SARS-CoV-2 Viral Load in Upper Respiratory Specimens of Infected Patients. N Engl J Med. 2020;382(12):1177-9.

9. Vukkadala N, Qian ZJ, Holsinger FC, Patel ZM, Rosenthal E. COVID-19 and the otolaryngologist preliminary evidence-based review. Laryngoscope. 2020.

10. Jensen AR, Nellemann HM, Overgaard J. Tumor progression in waiting time for radiotherapy in head and neck cancer. Radiother Oncol. 2007;84(1):5-10.

11. Jang JY, Choi N, Ko YH et al. Treatment outcomes in metastatic and localized high-grade salivary gland cancer: high chance of cure with surgery and post-operative radiation in T1-2 N0 high-grade salivary gland cancer. BMC Cancer. 2018;18(1):672.

12. Organization WH. Coronavirus disease (COVID-19) Pandemic.

13. Wu Z, McGoogan JM. Characteristics of and Important Lessons From the Coronavirus Disease 2019 (COVID-19) Outbreak in China: Summary of a Report of 72314 Cases From the Chinese Center for Disease Control and Prevention. JAMA. 2020.

\section{Hosted file}

Figure_1_-_Workflow_of_tele-consultation_clinic_(1).docx available at https://authorea.com/users/ 312328/articles/444308-mitigation-of-head-and-neck-cancer-service-disruption-during-covid19-in-hong-kong-through-telehealth-and-multi-institution-collaboration

\section{Hosted file}

Figure_2_-_Stratification_of_Head_and_Neck_Cancer_Operations.docx available at https://authorea . com/users/312328/articles/444308-mitigation-of-head-and-neck-cancer-service-disruption-duringcovid-19-in-hong-kong-through-telehealth-and-multi-institution-collaboration 